\title{
NORMING POINTS AND UNIQUE MINIMALITY OF ORTHOGONAL PROJECTIONS
}

BORIS SHEKHTMAN AND LESŁAW SKRZYPEK

Received 5 March 2005; Accepted 6 April 2005

We study the norming points and norming functionals of symmetric operators on $L_{p}$ spaces for $p=2 m$ or $p=2 m /(2 m-1)$. We prove some general result relating uniqueness of minimal projection to the set of norming functionals. As a main application, we obtain that the Fourier projection onto $\operatorname{span}[1, \sin x, \cos x]$ is a unique minimal projection in $L_{p}$.

Copyright (c) 2006 B. Shekhtman and L. Skrzypek. This is an open access article distributed under the Creative Commons Attribution License, which permits unrestricted use, distribution, and reproduction in any medium, provided the original work is properly cited.

\section{Introduction}

This paper had been motivated by the question of uniqueness of minimal projections in $L_{p}$ spaces. As an example, let $L_{p}(-\pi, \pi)$ be the Banach space of all $2 \pi$-periodic functions $f(t)$ such that

$$
\|f\|_{p}^{p}=\int|f|^{p} d \mu<\infty
$$

where $1 \leq p \leq \infty$ and $\mu$ is normalized Lebesgue measure on $(-\pi, \pi)$. Let $V$ be the space of trigonometric polynomials of degree $n$ and let $P$ be the Fourier projection from $L_{p}(-\pi, \pi)$ onto $V$. It is well known (see $[2,3,16])$ that $P$ is a minimal projection, that is, $P$ has the least norm among all projections from $L_{p}(-\pi, \pi)$ onto $V$. For $p=1,2$ and $p=\infty$, it was proved that $P$ is a unique projection that has this property (see $[4,7]$ ). For the other values of $p$, the uniqueness of the minimal projection is a wide open question.

More generally, let $V$ be a complemented subspace of $L_{p}(\mu)$. Define

$$
\lambda\left(V, L_{p}(\mu)\right)=\inf \left\{\|P\|: P \text { is a projection from } L_{p}(-\pi, \pi) \text { onto } V\right\}
$$

A projection $P$ from $L_{p}(-\pi, \pi)$ onto $V$ is called minimal if $\|P\|=\lambda\left(V, L_{p}(\mu)\right)$. The study 
of minimal projections have been instigated by Cheney and Price and continued by many authors (see $[2,3,5,8,10-13,15,16]$ ).

Yet, as of today the authors of this article do not know a single example of a subspace $V \subset L_{p}(\mu)$ with $1<p<\infty$ for which the minimal projection is not unique! There are a few (very few) instances for which the uniqueness of the minimal projection had been verified (see $[1,6,9,14,16-18,20])$. As an application of the general technique developed in this paper we will add a few bits and pieces to the list of examples of uniqueness of minimal projections. In particular we will prove that the minimal projection from $L_{p}(-\pi, \pi)$ onto $\operatorname{span}\{1, \sin t, \cos t\}$ is unique.

It was clear from the very beginning (see $[14,18,20]$ ) that the understanding of minimal projections depends strongly on our knowledge of norming points; that is, the functions $f \in L_{p}(\mu)$ such that $\|P f\|=\|P\|\|f\|$. By predicting the norming points of a minimal projection, one can find the minimal projections itself and forecast its properties. The spaces $L_{1}(\mu)$ have few extreme points and thus have few candidates for the norming points. This is one of the reasons for the successful studies of the minimal projections in $L_{1}(\mu)$. The space $L_{p}(\mu)$ on the other hand is a very different story. Every point of the unite ball of $L_{p}(\mu)$ is an extreme point. Even if the operator $P$ is given in advance, the norm of the operator $P: L_{p}(\mu) \rightarrow L_{p}(\mu)$ as well as its norming points are hard to come by. Readers, sceptical of our last statement are invited to find the norm of a nontrivial $3 \times 3$ matrix as an operator on $l_{p}^{3}$ for $1<p<\infty$ and $p \neq 2$.

In the next section, we will develop a convenient relationship between the symmetric operators on $L_{p}(\mu)$ and its norming points. In the last section we will apply these relationships to investigate the uniqueness of minimal projections. We will use the rest of this section to establish some notations.

The symbol $L_{p}(\mu)$ will always denote the real Banach space with an arbitrary positive Borel measure $\mu$. The index $p$, throughout this paper, will be of the form

$$
p=2 m \quad \text { or } \quad p=\frac{2 m}{2 m-1} \quad \text { with } m \in \mathbb{N} \text {. }
$$

The dual space to $L_{p}(\mu)$ is identified with $L_{q}(\mu)$ with $1 / p+1 / q=1$. For $(f, g) \in L_{p}(\mu) \times$ $L_{q}(\mu)$, the pairing $(f, g) \rightarrow \int f g d \mu$ defines the duality.

Definition 1.1. For an operator $P$ from a Banach space $X$ onto its subspace $Y$, define the set of norming points as

$$
\begin{gathered}
\mathfrak{N}(P):=\{x \in X:\|P x\|=\|P\|\|x\|, x \neq 0\} ; \\
\mathfrak{N}_{1}(P)=\{x \in \mathfrak{N}(P) ;\|x\|=1\}
\end{gathered}
$$

and norming functionals

$$
\begin{gathered}
\mathfrak{N F}(P):=\left\{f \in X^{*}:\|f \circ P\|=\|P\|\|f\|, f \neq 0\right\} \\
\mathfrak{N F}_{1}(P)=\{f \in \mathfrak{N F}(P) ;\|f\|=1\} .
\end{gathered}
$$


It is well known (see [16]) that if $P$ is compact, then $\mathfrak{N F}(P) \neq \varnothing$ if we additionally know that $X$ is reflexive then also $\mathfrak{N}(P) \neq \varnothing$ (without reflexivity, the last statement is not trueFourier projection $\mathscr{C}(\pi, \pi) \rightarrow \mathfrak{T}_{n}$ does not have a norming point). For $x \in X$, we let

$$
\begin{gathered}
\mathfrak{N}(x):=\left\{f \in X^{*}:\|f x\|=\|f\|\|x\|, f \neq 0\right\} \\
\mathfrak{N}_{1}(x)=\{f \in \mathfrak{N}(P) ;\|f\|=1\} .
\end{gathered}
$$

We also define the sets of norming pairs

$$
\begin{gathered}
\mathfrak{E}(P):=\left\{(f, x) \in X^{*} \times X: f(P x)=\|P\|\|x\|\|f\|,\|x\|\|f\| \neq 0\right\}, \\
\mathfrak{E}_{1}(P):=\{(f, x) \in \mathfrak{E}(P):\|x\|=\|f\|=1\} .
\end{gathered}
$$

The sets of norming pairs had been also termed extremal pairs by Chalmers and Metcalf (see $[2,3])$. The Holder inequality establishes an easy relation between norming points and norming pairs. This relationship is especially transparent in cases $p=2 \mathrm{~m} /$ $(2 m-1)$ or $p=2 m$ for some integer $m$. From now on, we will only deal with these values for $p$. To formalize it, we avail ourself of Holder functionals.

Definition 1.2. Let $f$ be a nonzero function in $L_{p}$. Define

$$
H_{p}(f)=\frac{(f)^{p-1}}{\left\|(f)^{p-1}\right\|_{q}} \in L_{q} .
$$

Observe that $H_{p}: L_{p} \backslash\{0\} \rightarrow L_{q}$ is a nonlinear (continuous) mapping. Here are a few simple properties of this functional.

Proposition 1.3. Let $p=2 m /(2 m-1)$ or $p=2 m$ for some integer $m$. Then

(1) $\left\|H_{p}(f)\right\|_{q}=1$,

(2) $\int f H_{p}(f) d \mu=\|f\|_{p}$,

(3) $H_{p}(\lambda f)=\operatorname{sign}(\lambda) H_{p}(f)$,

(4) $g \in \mathfrak{N}(f)$ if and only if $f \in \mathfrak{N}(g)$,

(5) if $0 \neq f \in L_{p}$, then $g \in \mathfrak{N}(f)$ if and only if $g=H_{p}(f)$.

\section{Orthogonal projections onto subspaces of $L_{p}$}

Let $V$ be a finite-dimensional subspace of $L_{p} \cap L_{2}$. By the orthogonal projection from $L_{p}$ onto $V$ we mean an orthogonal projection $P$ from $L_{2}$ onto $V$ considered as the mapping on $L_{p}$. In the above settings, $P^{*}$ is an orthogonal projection in $L_{q}$ with the range $V$ (regarded as a subspace of $L_{q}$ ). The following proposition is obvious.

Proposition 2.1. Let $V$ be a finite-dimensional subspace of $L_{p}$ and let $P$ be a projection from $L_{p}$ onto $V$. Regard $P$ as an operator from $L_{p}$ to $L_{p}$. Then $P^{*}$ is a projection regarded as an operator from $L_{q}$ to $L_{q}$. There exists

$$
\|P\|_{L_{p} \rightarrow L_{p}}=\left\|P^{*}\right\|_{L_{q} \rightarrow L_{q}}
$$


4 Norming points of orthogonal projections

Furthermore, the following are equivalent:

(1) $0 \neq(g, f) \in \mathfrak{E}_{*}(P)$,

(2) $0 \neq(f, g) \in \mathfrak{E}_{*}\left(P^{*}\right)$,

(3) $g \in \mathfrak{N}_{*}(P f)$ and $f \in \mathfrak{N}_{*}(P)$,

(4) $f \in \mathfrak{N}_{*}\left(P^{*} g\right)$ and $g \in \mathfrak{N}_{*}\left(P^{*}\right)$,

where $\mathfrak{N}_{*}$ (resp., $\left.\mathfrak{E}_{*}\right)$ stands for either $\mathfrak{N}$ or $\mathfrak{N}_{1}$ (resp., $\mathfrak{E}$ or $\left.\mathfrak{E}_{1}\right)$.

Keeping in mind the form of the Holder functional $H_{p}$, we obtain the following theorem.

Theorem 2.2. Let $p=2 m /(2 m-1)$ or $p=2 m$ and let $P$ be a projection from $L_{p}$ onto $V$. Suppose that $f \neq 0$. Then

$$
f \in \mathfrak{N}(P) \text { iff }(\|P\|)^{p} f^{p-1}=P^{*}\left((P f)^{p-1}\right) .
$$

In particular if $p=2 m$,

$$
f \in \mathfrak{N}(P) \text { iff }(\|P\|)^{2 m /(2 m-1)} f=P^{*}\left((P f)^{2 m-1}\right)^{1 /(2 m-1)},
$$

and if $p=2 m /(2 m-1)$,

$$
f \in \mathfrak{N}(P) \text { iff }(\|P\|)^{2 m} f=P^{*}\left((P f)^{1 /(2 m-1)}\right)^{2 m-1} .
$$

Proof. Without loss, we can assume that $f \in \mathfrak{N}_{1}(P)$. Take $g \in L_{q}$ such that $g P f=\|P\|$. So $g \in \mathfrak{N}_{1}(P f)$ and as a result from Proposition 1.3 we have $g=H_{p}(P f)$. Therefore $\left(H_{p}(P f), f\right) \in \mathfrak{E}_{1}(P)$. Using Proposition 2.1, we have $\left(f, H_{p}(P f)\right) \in \mathfrak{E}_{1}\left(P^{*}\right)$. This implies that

$$
\begin{aligned}
\|P\| & =\left\|P^{*}\right\|=\left\|P^{*}\left(H_{p}(P f)\right)\right\|_{q}=\left\|P^{*}\left(\frac{(P f)^{p-1}}{\left\|(P f)^{p-1}\right\|_{q}}\right)\right\|_{q} \\
& =\frac{P^{*}\left((P f)^{p-1}\right)}{\left\|(P f)^{p-1}\right\|_{q}}=\frac{\left\|P^{*}\left((P f)^{p-1}\right)\right\|_{q}}{\left(\|P f\|_{p}\right)^{p / q}}=\frac{\left\|P^{*}\left((P f)^{p-1}\right)\right\|_{q}}{\|P\|^{p / q}},
\end{aligned}
$$

hence

$$
\begin{gathered}
\left\|P^{*}\left((P f)^{p-1}\right)\right\|_{q}=(\|P\|)^{p}, \\
f \in \mathfrak{N}_{1}\left(P^{*}\left(H_{p}(P f)\right)\right) .
\end{gathered}
$$

Using Proposition 1.3, the second equality (2.7) gives us

$$
f=H_{q}\left(P^{*}\left(H_{p}(P f)\right)\right) .
$$


Now applying (2.6) to (2.8), we obtain

$$
\begin{aligned}
f & =H_{q}\left(P^{*}\left(H_{p}(P f)\right)\right)=H_{q}\left(P^{*}\left(\frac{(P f)^{p-1}}{\left\|(P f)^{p-1}\right\|_{q}}\right)\right) \\
& =H_{q}\left(\frac{P^{*}\left((P f)^{p-1}\right)}{\left\|(P f)^{p-1}\right\|_{q}}\right)=H_{q}\left(P^{*}\left((P f)^{p-1}\right)\right) \\
& =\frac{\left(P^{*}\left((P f)^{p-1}\right)\right)^{q-1}}{\left.\|\left(P^{*}((P f))^{p-1}\right)\right)^{q-1} \|_{p}}=\frac{\left(P^{*}\left((P f)^{p-1}\right)\right)^{q-1}}{\left(\left\|P^{*}\left((P f)^{p-1}\right)\right\|_{q}\right)^{q / p}}=\frac{\left(P^{*}\left((P f)^{p-1}\right)\right)^{q-1}}{(\|P\|)^{q}} .
\end{aligned}
$$

Multiplying and raising both sides to the power $1 /(q-1)=p-1$ gives (2.2).

Assume now that $f$ fulfills (2.2). Without loss, we may assume that $\|f\|_{p}=1$ and compute

$$
1=\int f^{p} d \mu=\int f f^{p-1} d \mu=\int \frac{f P^{*}\left((P f)^{p-1}\right)}{(\|P\|)^{p}} d \mu .
$$

Hence

$$
f\left(P^{*}\left((P f)^{p-1}\right)\right)=(\|P\|)^{p}
$$

On the other hand,

$$
f\left(P^{*}\left((P f)^{p-1}\right)\right) \leq\|f\|_{p} \cdot\left\|P^{*}\right\| \cdot\left\|(P f)^{p-1}\right\|_{q}=\|P\| \cdot\left(\|P f\|_{p}\right)^{p-1} .
$$

If we combine (2.11) and (2.12), then we get

$$
\|P\| \leq\|P f\|_{p}
$$

hence $f \in N_{1}(P)$.

Even though Theorem 2.2 gives us an if and only if condition, it cannot be applied directly to find a norming functionals. In order to check the equality in condition (2.2) one has to know $\|P\|$. We may drop $\|P\|$ and formulate Theorem 2.2 as follows.

Corollary 2.3. Let $p=2 m /(2 m-1)$ or $p=2 m$ and let $P$ be a projection from $L_{p}$ onto $V$. Suppose that $f \neq 0$ and $f \in \mathfrak{N}(P)$. Then there is a constant $M$ such that

$$
f^{p-1}=M P^{*}\left((P f)^{p-1}\right) .
$$

In particular if $p=2 m$,

$$
f=M P^{*}\left((P f)^{2 m-1}\right)^{1 /(2 m-1)}
$$

and if $p=2 m /(2 m-1)$,

$$
f=M P^{*}\left((P f)^{1 /(2 m-1)}\right)^{2 m-1}
$$


If we now investigate (2.14) then for $M=0$, we obtain $f=0$ and for $M=(\|P\|)^{-P} \leq 1$, we obtain all norming points for $P$. The problem is there are many $M$ for which there is a solution to (2.14). Consider the Fourier projection $P: L_{2 m /(2 m-1)} \rightarrow \operatorname{span}[1, \cos t, \sin t]$. In Section 3, it is proved that a function $f=(\cos t)^{2 m-1}$ is not a norming point for $P$, yet it satisfies (2.16) for some $M<1$. On the other hand in general, Corollary 2.3 brings some extremely useful information on norming points.

Theorem 2.4. Let $P$ be a projection from $L_{p}$ onto $V$. Define

$$
V^{\alpha}:=\left\{f^{\alpha}: f \in V\right\}
$$

If $p=2 m /(2 m-1)$ for some integer $m$, then

$$
\mathfrak{N}(P) \subset V^{2 m-1} .
$$

If $p=2 m$ for some integer $m$, then

$$
\mathfrak{N}(P) \subset V^{1 /(2 m-1)} .
$$

The proof follows immediately from Corollary 2.3.

Corollary 2.5. Assuming in the last theorem that $m=1$, an obvious result that the orthogonal projection $P: L_{2} \rightarrow L_{2}$ has a norming point in $V$ is obtained and being a projection, it follows that $\|P\|=1$.

CoRollary 2.6. Let $\mathfrak{T}_{n}=\operatorname{span}[1, \cos k t, \sin k t]_{k=1}^{n}$. Let $Q$ be an orthogonal projection from $L_{p}$ onto $\mathfrak{T}_{n}$. If $p=2 m /(2 m-1)$ for some integer $m$, then

$$
\|Q\|_{L_{p} \rightarrow L_{p}}=\|Q\|_{\mathfrak{T}_{n(2 m-1)} \rightarrow \mathfrak{T}_{n}}
$$

In the language of projectional constants, we can reformulate this result as

$$
\lambda\left(\mathfrak{T}_{n}, L_{p}\right)=\lambda\left(\mathfrak{T}_{n}, \mathfrak{T}_{(2 m-1) n}\right) .
$$

In terms of norming points, we conclude that there exists a trigonometric polynomial $g$ of degree $(2 m-1) n$ which is a norming point for $Q$. In stark contrast to the last corollary, we have the following corollary.

Corollary 2.7. Let $p=2 m>2$ and let $P$ be the orthogonal projection from $L_{p}$ onto $\mathfrak{T}_{n}$. Then for every trigonometric polynomial (of any degree) $f$,

$$
\|P f\|<\|P\|\|f\| .
$$

Proof. Let us assume that there is a trigonometric polynomial of the exact degree $N$ such that $f \in \mathfrak{N}_{1}(P)$. Since $\|P\|>1$ we have $N>n$. According to (2.15) we have $f^{2 m-1} \in V=$ $\mathfrak{T}_{n}$. But $f^{2 m-1}$ is a trigonometric polynomial of the exact degree $N(2 m-1)$. But $N(2 m-$ 1) $>n$ which gives a contradiction. 
From the above, we may conclude that if $p=2 m$, then any norming point for $P$ (an orthogonal projection onto polynomials $\mathfrak{T}_{n}$ ) is not a polynomial.

Remark 2.8. While the norming points of $P$ are not trigonometric polynomials, the previous corollary shows that the norming functionals $g \in \mathfrak{N}_{1}(P f)$ with $f \in \mathfrak{N}_{1}(P)$ are polynomials of exact degree $(2 m-1) n$. Hence, whether $p=2 m$ or $p=2 m /(2 m-1)$, at least one component of the norming pair $(g, f) \in \mathfrak{E}(P)$ is a polynomial of exact degree $(2 m-1) n$.

We finish this section with one more application of this technique to the best approximation problem in $L_{p}$.

Theorem 2.9. Let $p=2 m /(2 m-1)$ and let $\mathfrak{T}_{n}^{c}=\operatorname{Im}(\operatorname{Id}-P)$. That is, $\mathfrak{T}_{n}^{c}$ is the closure in $L_{p}$ of trigonometric polynomials with frequencies larger than $n$. Then for every $f \in \mathfrak{T}_{n}$, the element $(\mathrm{Id}-\mathrm{P})\left(f^{2 m-1}\right)$ is the unique best approximation to $P\left(f^{2 m-1}\right)$ from $\mathfrak{T}_{n}^{c}$.

Proof. Consider

$$
H_{p}\left(f^{2 m-1}\right)=\frac{\left(f^{2 m-1}\right)^{p-1}}{\left\|(f)^{p-1}\right\|_{q}}=\frac{1}{\left\|(f)^{p-1}\right\|_{q}} f .
$$

Since $f \in \mathfrak{T}_{n}$, we conclude that

$$
H_{p}\left(f^{2 m-1}\right) \perp \mathfrak{T}_{n}^{c}
$$

Also, by the definition of the Holder functional,

$$
\begin{aligned}
\left\|P f^{2 m-1}-(I-P) f^{2 m-1}\right\|_{p} & =\left\|f^{2 m-1}\right\|_{p}=H_{p}\left(f^{2 m-1}\right)\left(f^{2 m-1}\right) \\
& =H_{p}\left(f^{2 m-1}\right)\left(P f^{2 m-1}-(I-P) f^{2 m-1}\right) .
\end{aligned}
$$

Properties (2.24) and (2.25) combined with the well-known criterium (see [19]) for the best approximation imply the conclusion of the theorem.

The above theorem is very useful obtaining many examples of best approximation in $L_{p}$ - what we need is to raise some elements to the appropriate power. Here is a simple illustration of the usefulness of this theorem.

Example 2.10. Let $f=(1+\cos x+\sin x)$. Then $f^{3}=(1+\cos x+\sin x)^{3}=4+(9 / 2) \cos x+$ $(9 / 2) \sin x+3 \sin 2 x-(1 / 2) \cos 3 x+(1 / 2) \sin 3 x$. Hence

$$
\left(3 \sin 2 x-\frac{1}{2} \cos 3 x+\frac{1}{2} \sin 3 x\right)
$$

is the best approximation to

$$
\left(4+\frac{9}{2} \cos x+\frac{9}{2} \sin x\right)
$$

from the space $\mathfrak{T}_{1}^{c}$ (trigonometric polynomials with frequencies larger 1 ) in the space $L_{4 / 3}$. 


\section{Applications}

We start with general theorem which will connect uniqueness of a given projection to its norming points. First we state the following theorem.

Theorem 3.1 (Rudin) [21, III.B.13]. Let X be a Banach space and $V$ a complemented subspace. Let $G$ be a compact group which acts as a group of linear operators on $X$ such that

(1) $T_{g}(x)$ is a continuous function of $g$, for every $x \in X$,

(2) $T_{g}(V) \subset V$, for all $g \in G$,

(3) $T_{g}$ are isometries, for all $g \in G$.

Furthermore, assume that there exists only one projection $P: X \rightarrow V$ which commutes with $G$. Then this projection is minimal.

Once we know that there is only one projection $P$ commuting with $G$, the following can be easily found: fix any projection $Q$ from $X$ onto $V$, then this projection $P$ equals

$$
P(x)=\int_{G} T_{g} Q T_{g^{-1}}(x) d g, \quad \text { for } x \in X .
$$

Now we are ready to prove the following theorem.

Theorem 3.2. Let $V$ be a complimented subspace of $L^{p}(\mu)(1<p<\infty)$ and assume there is a group $G$ acting on $L_{p}(\mu)$ as in Theorem 3.1. Let $P: L^{p}(\mu) \rightarrow V$ be a minimal projection given by (3.1). Assume additionally that the set of norming functionals for $P$ is total on $V$ (i.e., if $v \in V$, and $f(v)=0$ for any $f \in \mathfrak{N F}(P)$, then $v=0$ ). Then projection $P$ is the unique minimal projection.

Proof. Assume to the contrary that there is another minimal projection $Q$. Therefore $\|P\|=\|Q\|$. We will prove now that

$$
\mathfrak{E}_{1}(P) \subset \mathfrak{E}_{1}(Q)
$$

To do this assume to contrary that $\|f(Q x)\|<\|Q\|=\|P\|$. As in the proof of Theorem 3.1 observe that the function

$$
g \longmapsto T g(f) Q T g^{-1}(x)
$$

is continuous. Therefore in some open neighborhood $U$ of $g=1$, we have

$$
\left\|\operatorname{Tg}(f) Q T g^{-1}(x)\right\|<\|Q\|=\|P\|,
$$

and by (3.1)

$$
\begin{aligned}
\|P\| & =\|f(P x)\|=\left\|\int_{G} T_{g} f Q T_{g^{-1}}(x) d g\right\| \leq \int_{G}\left\|T_{g} f Q T_{g^{-1}}(x)\right\| d g \\
& =\int_{U}\left\|T_{g} f Q T_{g^{-1}}(x)\right\| d g+\int_{G \backslash U}\left\|T_{g} f Q T_{g^{-1}}(x)\right\| d g \\
& <\int_{U}\|Q\| d g+\int_{G \backslash U}\|Q\| d g=\|P\|,
\end{aligned}
$$


which is a contradiction. Now (3.2) gives us

$$
f(P)(x)=f(Q)(x)=\|P\|=\|Q\|, \quad \text { for any }(f, x) \in \mathfrak{E}_{1}(P) .
$$

That is

$$
\left(\frac{P^{*} f}{\|P\|}\right)(x)=\left(\frac{Q^{*} f}{\|Q\|}\right)(x)=1, \quad \text { for any }(f, x) \in \mathfrak{E}_{1}(P) .
$$

But $L_{p}$ is a smooth space - an element $x$ can only have one norming functional, therefore

$$
P^{*} f=Q^{*} f, \quad \text { for any } f \in \mathfrak{N}_{1}(P) \text {. }
$$

Take any $x \in L_{p}(\mu)$, we have $P x-Q x \in V$. Now for any $f \in N_{1}(P)$ using (3.8), we have

$$
f(P x-Q x)=\left(P^{*} f-Q^{*} f\right) x=0,
$$

but the set of norming functionals is total on $V$ so $P x=Q x$, hence $P=Q$.

We will prove now that for $p=2 m$ or $p=2 m /(2 m-1)$ orthogonal projection onto $\operatorname{span}[1, \cos t, \sin t]$ is unique minimal projection. The main trick is based on Corollary 2.3. For Fourier projection $P$, we have $P^{*}=P$. It implies that if $f$ is a norming point for $P$ (for $p=2 m /(2 m-1)$ ), then for some constant $M<\leq 1$ we have

$$
f=M\left(P\left((P f)^{1 /(2 m-1)}\right)\right)^{2 m-1} .
$$

Hence any norming point has to be of the form

$$
\begin{gathered}
f=g^{2 m-1}, \quad g \in \operatorname{span}[1, \cos t, \sin t], \\
g=\operatorname{MP}\left(\left(P\left(g^{2 m-1}\right)\right)^{1 /(2 m-1)}\right),
\end{gathered}
$$

for some constant $M$. As we will see later, both (3.10) and (3.11) are only necessary conditions, $g=\cos t$ and $f=(\cos t)^{2 m-1}$ fulfill, respectively, (3.11) and (3.10), yet $f=(\cos t)^{2 m-1}$ is not a norming point for a Fourier projection. That is actually the main difficulty in showing uniqueness of Fourier projection.

Theorem 3.3. Let $\mathfrak{T}_{1}=\operatorname{span}[1, \cos t, \sin t]$. Let $P$ be an orthogonal projection from $L_{p}$ onto $\mathfrak{T}_{1}$. If $p=2 m /(2 m-1)$ for some integer $m>1$, then there is a norming pair $(g, f)$ for $P$ such that

$$
\begin{aligned}
& \left.f\right|_{\operatorname{span}[1, \cos t, \sin t]}=a_{0}+a_{1} \cos t+a_{2} \sin t, \\
& \left.g\right|_{\operatorname{span}[1, \cos t, \sin t]}=b_{0}+b_{1} \cos t+b_{2} \sin t,
\end{aligned}
$$

where $a_{0}, a_{1}, a_{2}, b_{0}, b_{1}, b_{2} \neq 0$. 
Proof. Fourier projection is invariant under the isometries $I_{\theta}: f(t) \mapsto f(t+\theta)$, (i.e., $P I_{\theta}=$ $\left.I_{\theta} P\right)$-see $[2,3,16]$. This implies

$$
f(t) \in \mathfrak{N}(P) \Longleftrightarrow f(t+\theta) \in \mathfrak{N}(P)
$$

for any $\theta$. So assume that $f$ is a norming point for $P$. According to (3.11),

$$
f=\left(a_{0}+a_{1} \cos t+a_{2} \sin t\right)^{2 m-1} .
$$

Observe that $a_{1}^{2}+a_{2}^{2}>0$ (otherwise $\|P\|=1$ ), therefore

$$
\begin{aligned}
f & =\left(a_{0}+a_{1} \cos t+a_{2} \sin t\right)^{2 m-1} \\
& =\left(a_{1}^{2}+a_{2}^{2}\right)^{(2 m-1) / 2}\left(\frac{a_{0}}{\sqrt{a_{1}^{2}+a_{2}^{2}}}+\frac{a_{1}}{\sqrt{a_{1}^{2}+a_{2}^{2}}} \cos t+\frac{a_{2}}{\sqrt{a_{1}^{2}+a_{2}^{2}}} \sin t\right)^{2 m-1} .
\end{aligned}
$$

Now there is $\theta$ such that $\cos (\theta)=a_{1} /\left(\sqrt{a_{1}^{2}+a_{2}^{2}}\right)$ and $\sin (\theta)=a_{2} /\left(\sqrt{a_{1}^{2}+a_{2}^{2}}\right)$, and now

$$
\begin{aligned}
f & =\left(a_{0}+a_{1} \cos t+a_{2} \sin t\right)^{2 m-1} \\
& =\left(a_{1}^{2}+a_{2}^{2}\right)^{(2 m-1) / 2}\left(\frac{a_{0}}{\sqrt{a_{1}^{2}+a_{2}^{2}}}+\cos \theta \cos t+\sin \theta \sin t\right)^{2 m-1} \\
& =\left(a_{1}^{2}+a_{2}^{2}\right)^{(2 m-1) / 2}\left(\frac{a_{0}}{\sqrt{a_{1}^{2}+a_{2}^{2}}}+\cos (t-\theta)\right)^{2 m-1} .
\end{aligned}
$$

Using (3.13), it follows that in searching for norming points of $P$, we can restrict ourselves to the functions of the form

$$
f(t)=(x+\cos t)^{2 m-1}
$$

We will prove that $P$ has a norming point of the form (3.17) for some $x \neq 0$. To do this, we need to prove that the function

$$
T(x)=\frac{\left\|P\left((x+\cos t)^{2 m-1}\right)\right\|_{p}^{p}}{\left\|(x+\cos t)^{2 m-1}\right\|_{p}^{p}}=\frac{\int_{0}^{2 \pi}\left(P\left((x+\cos t)^{2 m-1}\right)\right)^{2 m /(2 m-1)} d t}{\int_{0}^{2 \pi}(x+\cos t)^{2 m} d t}
$$

does not attain its maximum at $x=0$. Define (whenever it makes sense)

$$
F(\nu)=\int_{0}^{2 \pi}(\cos t)^{\nu} d t
$$


Observe that for any $k \in \mathbb{N}$,

$$
F(2 k)=\int_{0}^{2 \pi}(\cos t)^{2 k} d t=4 \int_{0}^{\pi / 2}(\cos t)^{2 k} d t=\frac{2 \cdot \sqrt{\pi} \cdot \Gamma(1 / 2+k)}{\Gamma(1+k)}
$$

and by the symmetry of the cosine graph,

$$
F(2 k-1)=\int_{0}^{2 \pi}(\cos t)^{2 k-1}=0, \quad F\left(\frac{1}{2 k-1}\right)=\int_{0}^{2 \pi}(\cos t)^{1 / 2 k-1}=0 .
$$

What is more, for any $k \in \mathbb{N}$,

$$
\begin{aligned}
F\left(\frac{2 k}{2 k-1}\right) & =\int_{0}^{2 \pi}(\cos t)^{2 k /(2 k-1)} d t \\
& =4 \int_{0}^{\pi / 2}(\cos t)^{2 k /(2 k-1)} d t=\frac{2 \cdot \sqrt{\pi} \cdot \Gamma(1 / 2+k /(2 k-1))}{\Gamma(1+k /(2 k-1))}, \\
F\left(-\frac{2 k-2}{2 k-1}\right) & =\int_{0}^{2 \pi}\left(\frac{1}{\cos t}\right)^{(2 k-2) /(2 k-1)} d t \\
& =4 \int_{0}^{\pi / 2}\left(\frac{1}{\cos t}\right)^{(2 k-2) /(2 k-1)} d t=\frac{2 \cdot \sqrt{\pi} \cdot \Gamma(1 /(4 k-2))}{\Gamma(k /(2 k-1))} .
\end{aligned}
$$

First we need to compute $P\left((x+\cos t)^{2 m-1}\right)$. Observe that

$$
P\left((x+\cos t)^{2 m-1}\right)=a_{0}(x)+a_{1}(x) \cos t,
$$

where

$$
\begin{gathered}
a_{0}(x)=\frac{1}{2 \pi} \int_{0}^{2 \pi}(x+\cos t)^{2 m-1} d t, \\
a_{1}(x)=\frac{1}{2 \pi} \int_{0}^{2 \pi}(x+\cos t)^{2 m-1} \cos t d t .
\end{gathered}
$$

Using Newton formula $(x+\cos t)^{2 m-1}=\sum_{i=1}^{2 m-1}\left(\begin{array}{c}2 m-1 \\ i\end{array}\right) x^{i}(\cos t)^{2 m-1-i}$ and obtain

$$
\begin{aligned}
a_{0}(x) & =\frac{1}{2 \pi} \int_{0}^{2 \pi} \sum_{i=1}^{2 m-1}\left(\begin{array}{c}
2 m-1 \\
i
\end{array}\right) x^{i}(\cos t)^{2 m-1-i} d t \\
& =\sum_{i=1}^{2 m-1} \frac{1}{2 \pi}\left(\begin{array}{c}
2 m-1 \\
i
\end{array}\right) x^{i} \int_{0}^{2 \pi}(\cos t)^{2 m-1-i} d t \\
& =\sum_{i=1}^{2 m-1} \frac{1}{2 \pi}\left(\begin{array}{c}
2 m-1 \\
i
\end{array}\right) x^{i} F(2 m-1-i) \\
& =\sum_{i-\text { odd }} \frac{1}{2 \pi}\left(\begin{array}{c}
2 m-1 \\
i
\end{array}\right) x^{i} F(2 m-1-i),
\end{aligned}
$$


and similarly

$$
\begin{aligned}
a_{1}(x) & =\frac{1}{2 \pi} \int_{0}^{2 \pi} \sum_{i=1}^{2 m-1}\left(\begin{array}{c}
2 m-1 \\
i
\end{array}\right) x^{i}(\cos t)^{2 m-1-i} \cos t d t \\
& =\sum_{i=1}^{2 m-1} \frac{1}{2 \pi}\left(\begin{array}{c}
2 m-1 \\
i
\end{array}\right) x^{i} \int_{0}^{2 \pi}(\cos t)^{2 m-1-i} \cos t d t \\
& =\sum_{i=1}^{2 m-1} \frac{1}{2 \pi}\left(\begin{array}{c}
2 m-1 \\
i
\end{array}\right) x^{i} F(2 m-i) \\
& =\sum_{i-\text { even }} \frac{1}{2 \pi}\left(\begin{array}{c}
2 m-1 \\
i
\end{array}\right) x^{i} F(2 m-i) .
\end{aligned}
$$

We have $T(x)=H(x) / G(x)$, where

$$
\begin{gathered}
H(x)=\int_{0}^{2 \pi}\left(P\left((x+\cos t)^{2 m-1}\right)\right)^{2 m /(2 m-1)} d t=\int_{0}^{2 \pi}\left(a_{0}(x)+a_{1}(x) \cos t\right)^{2 m /(2 m-1)} d t \\
G(x)=\int_{0}^{2 \pi}(x+\cos t)^{2 m} d t .
\end{gathered}
$$

We need to prove that

$$
\frac{H(x)}{G(x)}>\frac{H(0)}{G(0)}
$$

for some small $x>0$. Since $H(0), G(0)>0$, this is equivalent to showing that the function $q(x)=H(x) G(0)-G(x) H(0)$ is increasing for some small $x>0$. We will prove that by showing that $q^{\prime \prime}(0)>0$ (interestingly enough, one may easily show that $q^{\prime}(0)=0$ so $q(x)$ as well as $T(x)$, has in fact local minimum at zero).

We will compute $G(0), G^{\prime \prime}(0), H(0), H^{\prime \prime}(0)$ next. To do so, observe that

$$
\begin{gathered}
a_{0}(x)=\left(\frac{1}{2 \pi}\left(\begin{array}{c}
2 m-1 \\
1
\end{array}\right) F(2 m-2)\right) x+\left(\frac{1}{2 \pi}\left(\begin{array}{c}
2 m-1 \\
3
\end{array}\right) F(2 m-4)\right) x^{3}+\cdots \\
a_{1}(x)=\left(\frac{1}{2 \pi} F(2 m)\right)+\left(\frac{1}{2 \pi}\left(\begin{array}{c}
2 m-1 \\
2
\end{array}\right) F(2 m-2)\right) x^{2}+\cdots
\end{gathered}
$$

therefore

$$
\begin{aligned}
a_{0}(0)=0, & \left(a_{0}\right)^{\prime}(0)=\frac{1}{2 \pi}(2 m-1) F(2 m-2), \quad\left(a_{0}\right)^{\prime \prime}(0)=0, \\
a_{1}(0)=\frac{1}{2 \pi} F(2 m), & \left(a_{1}\right)^{\prime}(0)=0, \quad\left(a_{1}\right)^{\prime \prime}(0)=\frac{1}{2 \pi}(2 m-1)(2 m-2) F(2 m-2) .
\end{aligned}
$$


Now

$$
\begin{gathered}
G(0)=\int_{0}^{2 \pi}(\cos t)^{2 m} d t=F(2 m), \\
G^{\prime \prime}(x)=\int_{0}^{2 \pi} 2 m(2 m-1)(x+\cos t)^{2 m-2} d t .
\end{gathered}
$$

Therefore

$$
G^{\prime \prime}(0)=2 m(2 m-1) F(2 m-2) .
$$

Also

$$
\begin{aligned}
H(0) & =\int_{0}^{2 \pi}\left(a_{0}(0)+a_{1}(0) \cos t\right)^{2 m /(2 m-1)} d t \\
& =\int_{0}^{2 \pi}\left(\frac{1}{2 \pi} F(2 m) \cos t\right)^{2 m /(2 m-1)} d t \\
& =\left(\frac{1}{2 \pi}\right)^{2 m /(2 m-1)}(F(2 m))^{2 m /(2 m-1)} F\left(\frac{2 m}{2 m-1}\right),
\end{aligned}
$$

and lastly we will compute $H^{\prime \prime}(0)$,

$$
\begin{aligned}
H^{\prime \prime}(x)= & \left(\int_{0}^{2 \pi}\left(a_{0}(x)+a_{1}(x) \cos t\right)^{2 m /(2 m-1)} d t\right)^{\prime \prime}(x) \\
= & \frac{2 m}{(2 m-1)^{2}} \int_{0}^{2 \pi}\left(a_{0}(x)+a_{1}(x) \cos t\right)^{-(2 m-2) /(2 m-1)}\left(a_{0}^{\prime}(x)+a_{1}^{\prime}(x) \cos t\right)^{2} d t \\
& +\frac{2 m}{2 m-1} \int_{0}^{2 \pi}\left(a_{0}(x)+a_{1}(x) \cos t\right)^{1 /(2 m-1)}\left(a_{0}^{\prime \prime}(x)+a_{1}^{\prime \prime}(x) \cos t\right) d t
\end{aligned}
$$

As a result,

$$
\begin{aligned}
H^{\prime \prime}(0)= & \frac{2 m}{(2 m-1)^{2}} \int_{0}^{2 \pi}\left(\frac{1}{2 \pi} F(2 m) \cos t\right)^{-(2 m-2) /(2 m-1)}\left(\frac{1}{2 \pi}(2 m-1) F(2 m-2)\right)^{2} d t \\
& +\frac{2 m}{2 m-1} \int_{0}^{2 \pi}\left(\frac{1}{2 \pi} F(2 m) \cos t\right)^{1 /(2 m-1)}\left(\frac{1}{2 \pi}(2 m-1)(2 m-2) F(2 m-2) \cos t\right) d t \\
= & \frac{2 m}{(2 m-1)^{2}}\left(\frac{1}{2 \pi} F(2 m)\right)^{-(2 m-2) /(2 m-1)}\left(\frac{1}{2 \pi}(2 m-1) F(2 m-2)\right)^{2} F\left(-\frac{2 m-2}{2 m-1}\right) \\
& +\frac{2 m}{2 m-1}\left(\frac{1}{2 \pi} F(2 m)\right)^{1 /(2 m-1)} \frac{1}{2 \pi}(2 m-1)(2 m-2) F(2 m-2) F\left(\frac{2 m}{2 m-1}\right) \\
= & \left(\frac{1}{2 \pi}\right)^{2 m /(2 m-1)} 2 m(F(2 m))^{-(2 m-2) /(2 m-1)}(F(2 m-2))^{2} F\left(-\frac{2 m-2}{2 m-1}\right) \\
& +\left(\frac{1}{2 \pi}\right)^{2 m /(2 m-1)} 2 m(2 m-2)(F(2 m))^{1 /(2 m-1)} F(2 m-2) F\left(\frac{2 m}{2 m-1}\right) .
\end{aligned}
$$


Combining what we have computed, we may state that $H^{\prime \prime}(0) G(0)-G^{\prime \prime}(0) H(0)>0$ is equivalent to

$$
\begin{aligned}
& \left(\frac{1}{2 \pi}\right)^{2 m /(2 m-1)} 2 m(F(2 m))^{1 /(2 m-1)}(F(2 m-2))^{2} F\left(-\frac{2 m-2}{2 m-1}\right) \\
& +\left(\frac{1}{2 \pi}\right)^{2 m /(2 m-1)} 2 m(2 m-2)(F(2 m))^{2 m /(2 m-1)} F(2 m-2) F\left(\frac{2 m}{2 m-1}\right) \\
& >\left(\frac{1}{2 \pi}\right)^{2 m /(2 m-1)} 2 m(2 m-1)(F(2 m))^{2 m /(2 m-1)} F(2 m-2) F\left(\frac{2 m}{2 m-1}\right),
\end{aligned}
$$

which after simplifications turns out to be

$$
F(2 m-2) F\left(-\frac{2 m-2}{2 m-1}\right)>F(2 m) F\left(\frac{2 m}{2 m-1}\right) .
$$

We may observe that $F(2 x)>F(2 x+2)$ as follows. Since $1>\cos ^{2}(t)$, we have

$$
F(2 x)=4 \int_{0}^{\pi / 4}(\cos (t))^{2 x-2} d t>4 \int_{0}^{\pi / 4}(\cos (t))^{2 x-2}(\cos (t))^{2} d t=F(2 x+2) .
$$

Letting now $2 x=2 m-2$, we get $F(2 m-2)>F(2 m)$ and letting $x=-(2 m-2) /(2 m-1)$, we get $F(-(2 m-2) /(2 m-1))>F(2 m /(2 m-1))$. These two inequalities combined together gives (3.37).

Therefore, we have just shown that there is a norming point for $P$ of the form

$$
f(t)=(x+\cos t)^{2 m-1}
$$

for some $x \neq 0$. Now according to (3.23),

$$
\left.f\right|_{\operatorname{span}[1, \cos t, \sin t]}=a_{0}(x)+a_{1}(x) \cos t
$$

where $a_{0}(x), a_{1}(x)$ are given by (3.25) and (3.26). Observe that

$$
\begin{gathered}
a_{0}(x)=x\left(\sum_{i=1}^{m-1} p_{i} x^{2 i}\right), \quad p_{i}>0 \\
a_{1}(x)=\sum_{i=1}^{m-1} q_{i} x^{2 i}, \quad q_{i}>0,
\end{gathered}
$$

and since $x \neq 0$ it gives us $a_{0}(x) \neq 0$ and $a_{1}(x) \neq 0$. Let $g$ be the norming functional associated with $f$ (i.e., $\left.(f, g) \in \mathfrak{E}_{1}(P)\right)$. Using (3.40) for some constant $K$, we have

$$
P f=K(y+\cos t), \quad y \neq 0 .
$$

Moreover $g=\mathfrak{N}(P f)$ and using Proposition 1.3 for some constant $L$, we have

$$
g=L(y+\cos t)^{1 /(2 m-1)} .
$$


We may write

$$
\left.g\right|_{\operatorname{span}[1, \cos t, \sin t]}=b_{0}(y)+b_{1}(y) \cos t+b_{2}(y) \sin t,
$$

and then we will show that $b_{0}(y)$. To do so, observe that

$$
b_{0}(y)=L \int_{0}^{2} \pi g(t) d t=2 L \int_{0}^{\pi} g(t) d t=2 L \int_{0}^{\pi}(y+\cos t)^{1 /(2 m-1)} d t .
$$

Without loss of generality, we may assume that $y>0$. If $y \geq 1$, then the function inside the integral is $\geq 0$ and then since $y>0$, it follows that $b_{0}(y)>0$. If $y \in(0,1)$, then there exists $t_{0} \in(0, \pi / 2)$ such that $y=\cos \left(\pi / 2-t_{0}\right)$, and we can make some estimations:

$$
\begin{aligned}
\int_{0}^{\pi}(y+\cos t)^{1 /(2 m-1)} d t= & \int_{0}^{\pi / 2+t_{0}}(y+\cos t)^{1 /(2 m-1)} d t+\int_{\pi / 2-t_{0}}^{\pi}(y+\cos t)^{1 /(2 m-1)} d t \\
= & \int_{0}^{\pi / 2+t_{0}}(y+\cos t)^{1 /(2 m-1)} d t+\int_{0}^{\pi / 2-t_{0}}(y-\cos t)^{1 /(2 m-1)} d t \\
= & \int_{0}^{\pi / 2-t_{0}}\left((y+\cos t)^{1 /(2 m-1)}-(y-\cos t)^{1 /(2 m-1)}\right) d t \\
& +\int_{\pi / 2-t_{0}}^{\pi / 2+t_{0}}(y+\cos t)^{1 /(2 m-1)} d t \geq \int_{\pi / 2-t_{0}}^{\pi / 2+t_{0}}(y+\cos t)^{1 /(2 m-1)} d t>0 .
\end{aligned}
$$

This proves that $b_{0}(y) \neq 0$. It is easy to prove that $b_{1}(y)^{2}+b_{2}(y)^{2}>0$. If not, then $P g=b$, where $b$ is a constant function. But $f(P g)=\|P\|$ in $L_{2 m}$ and as a result of Proposition 1.3 for some constant $C$, we would have $f=C(b)^{2 m-1}$ which implies that $\|P\|=1$, a contradiction. Since we know that in (3.40) $a_{0}(x), a_{1}(x) \neq 0$ and in (3.44) $b_{0}(y) \neq 0$ and one of $b_{1}(y), b_{2}(y)$ is $\neq 0$, we may apply (3.13) to obtain the result.

Theorem 3.4. Let $\mathfrak{T}_{1}=\operatorname{span}[1, \cos t, \sin t]$. Let $P$ be an orthogonal projection from $L_{p}$ onto $\mathfrak{T}_{1}$. If $p=2 m$ for some integer $m>1$, then there is a norming pair $(g, f)$ for $P$ such that

$$
\begin{aligned}
& \left.f\right|_{\operatorname{span}[1, \cos t, \sin t]}=a_{0}+a_{1} \cos t+a_{2} \sin t, \\
& \left.g\right|_{\operatorname{span}[1, \cos t, \sin t]}=b_{0}+b_{1} \cos t+b_{2} \sin t,
\end{aligned}
$$

where $a_{0}, a_{1}, a_{2}, b_{0}, b_{1}, b_{2} \neq 0$.

The proof is a straightforward consequence of Theorem 3.3 and Proposition 2.1.

Theorem 3.5. Let $\mathfrak{T}_{1}=\operatorname{span}[1, \cos t, \sin t]$. Let $P$ be the orthogonal projection from $L_{p}$ onto $\mathfrak{T}_{1}$. If $p=2 m /(2 m-1)$ or $p=2 m$ for some integer $m>1$, then $P$ is a unique minimal projection.

Proof. Having Theorems 3.3 and 3.4, we may apply Theorem 3.2 to obtain uniqueness of the orthogonal projection $P$.

Observe that, due to (3.13), Fourier projection always has infinite many norming points and norming functionals. Therefore, Corollary 2.6 produces us an extremely interesting example. 
Remark 3.6. There is a finite dimensional smooth space $X$ and its subspace $V$ such that the minimal projection from $X$ onto $V$ has norm greater than one and it has infinitely many norming points and norming functionals.

The above example shows us the crucial difference between the study of minimal projections in $\ell_{p}$ and $\ell_{1}$ spaces.

\section{References}

[1] M. Baronti and P. L. Papini, Norm-one projections onto subspaces of $l_{p}$, Annali di Matematica Pura ed Applicata. Serie Quarta 152 (1988), 53-61.

[2] B. L. Chalmers and F. T. Metcalf, The determination of minimal projections and extensions in $L^{1}$, Transactions of the American Mathematical Society 329 (1992), no. 1, 289-305.

[3] _ A characterization and equations for minimal projections and extensions, Journal of Operator Theory 32 (1994), no. 1, 31-46.

[4] E. W. Cheney, C. R. Hobby, P. D. Morris, F. Schurer, and D. E. Wulbert, On the minimal property of the Fourier projection, Transactions of the American Mathematical Society 143 (1969), 249258.

[5] E. W. Cheney and K. H. Price, Minimal Projections in, Approximation Theory, in Proceedings of Symp., Lancaster, July 1969 ( Talbot, ed.), Academic Press, London, 1970, pp. 261-289.

[6] H. B. Cohen and F. E. Sullivan, Projecting onto cycles in smooth, reflexive Banach spaces, Pacific Journal of Mathematics 34 (1970), 355-364.

[7] S. D. Fisher, P. D. Morris, and D. E. Wulbert, Unique minimality of Fourier projections, Transactions of the American Mathematical Society 265 (1981), no. 1, 235-246.

[8] C. Franchetti, Projections onto hyperplanes in Banach spaces, Journal of Approximation Theory 38 (1983), no. 4, 319-333.

[9] - The norm of the minimal projection onto hyperplanes in $L^{p}[0,1]$ and the radial constant, Unione Matematica Italiana. Bollettino. B. Serie VII 4 (1990), no. 4, 803-821.

[10] C. Franchetti and E. W. Cheney, Minimal projections in $L_{1}$-spaces, Duke Mathematical Journal 43 (1976), no. 3, 501-510.

[11] J. R. Isbell and Z. Semadeni, Projection constants and spaces of continuous functions, Transactions of the American Mathematical Society 107 (1963), no. 1, 38-48.

[12] H. König and N. Tomczak-Jaegermann, Norms of minimal projections, Journal of Functional Analysis 119 (1994), no. 2, 253-280.

[13] G. Lewicki, On the unique minimality of the Fourier-type extensions in $L_{1}$-space, Function Spaces (Poznań, 1998) (H. Hudzik and L. Skrzypczak, eds.), Lecture Notes in Pure and Appl. Math., no. 213, Dekker, New York, 2000, pp. 337-345.

[14] G. Lewicki and L. Skrzypek, Chalmers-Metcalf Operator and Uniqueness of Minimal Projections, IMUJ Preprint 2005/09.

[15] P. D. Morris and E. W. Cheney, On the existence and characterization of minimal projections, Journal $f$ ü r die Reine und Angewandte Mathematik 270 (1974), 61-76.

[16] W. Odyniec and G. Lewicki, Minimal Projections in Banach Spaces, Lecture Notes in Mathematics, vol. 1449, Springer, Berlin, 1990.

[17] S. Rolewicz, On projections on subspaces of codimension one, Polska Akademia Nauk. Instytut Matematyczny. Studia Mathematica 96 (1990), no. 1, 17-19.

[18] B. Shekhtman and L. Skrzypek, Uniqueness of minimal projections onto two-dimensional subspaces, Studia Mathematica 168 (2005), no. 3, 273-284.

[19] I. Singer, Best Approximation in Normed Linear Spaces by Elements of Linear Subspaces, Die Grundlehren der mathematischen Wissenschaften, vol. 171, Publishing House of the Academy of the Socialist Republic of Romania, Bucharest; Springer, New York, 1970. 
[20] L. Skrzypek, Uniqueness of minimal projections in smooth matrix spaces, Journal of Approximation Theory 107 (2000), no. 2, 315-336.

[21] P. Wojtaszczyk, Banach Spaces for Analysts, Cambridge Studies in Advanced Mathematics, vol. 25, Cambridge University Press, Cambridge, 1991.

Boris Shekhtman: Department of Mathematics, University of South Florida, 4202 E. Fowler Avenue, PHY 114 Tampa, FL 33620-5700, USA

E-mail address: boris@math.usf.edu

Lesław Skrzypek: Department of Mathematics, University of South Florida, 4202 E. Fowler Avenue, PHY 114 Tampa, FL 33620-5700, USA

E-mail address: skrzypek@math.usf.edu 


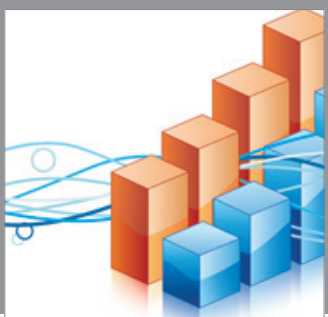

Advances in

Operations Research

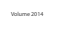



\section{The Scientific} World Journal
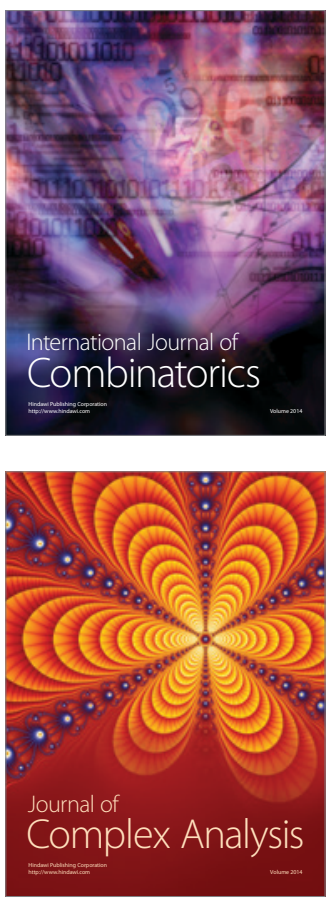

International Journal of

Mathematics and

Mathematical

Sciences
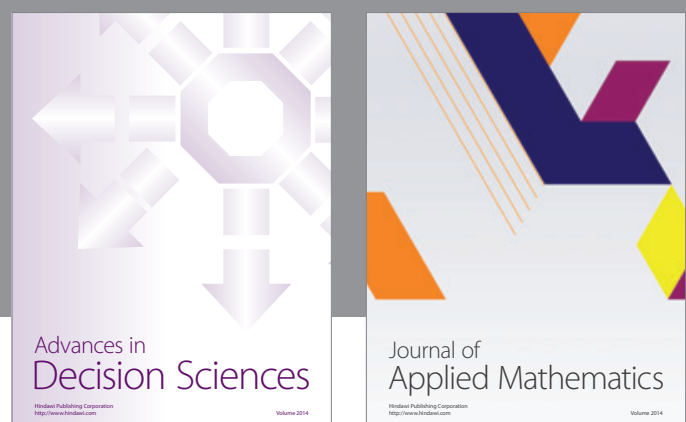

Journal of

Applied Mathematics


Submit your manuscripts at http://www.hindawi.com
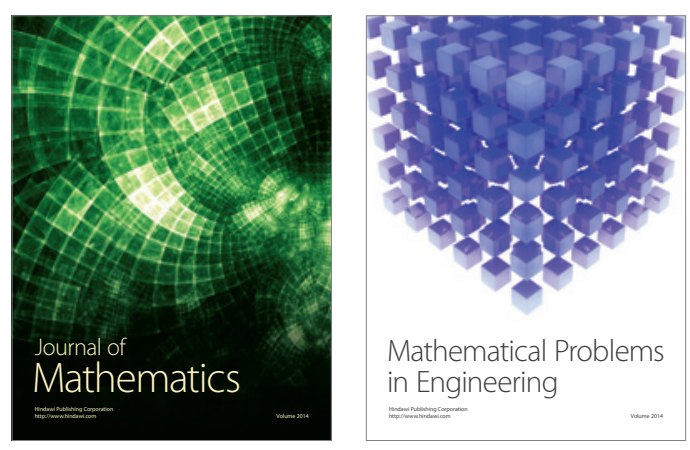

Mathematical Problems in Engineering
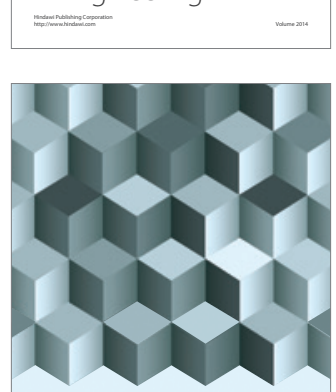

Journal of

Function Spaces
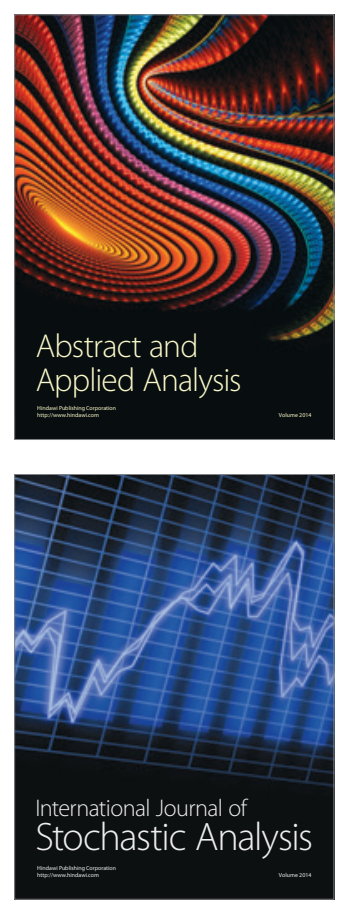

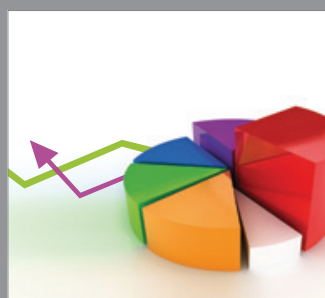

ournal of

Probability and Statistics

Promensencen
